\title{
Purification of single-stranded DNA by co-polymerization with acrylamide and electrophoresis
}

\author{
Tulsi Ram Damase ${ }^{1}$, Andrew D. Ellington ${ }^{2}$, and Peter B. Allen ${ }^{1}$ \\ ${ }^{1}$ University of Idaho, Department of Chemistry, Moscow, ID and '2University of Texas at Austin, \\ Department of Chemistry and Biochemistry, Institute for Cellular and Molecular Biology, Austin, TX
}

BioTechniques 62:275-282 (June 2017) doi 10.2144/000114557

Keywords: DNA; aptamer purification; single-strand generation

Supplementary material for this article is available at www.BioTechniques.com/article/114557.

Single-stranded DNA (ssDNA) oligonucleotides are useful as aptamers, hybridization probes and for emerging applications in DNA nanotechnology. Current methods to purify ssDNA require both a strand-separation step and a separate size-separation step but may still leave double-stranded DNA (dsDNA) impurities in the sample. Here, we use commercially available acrydite DNA primers to immobilize one strand of a PCR product within a polyacrylamide matrix. Electrophoresis moves the non-crosslinked DNA into the gel where the single-stranded product of desired size can be recovered. Our results show this method produces high yields of pure ssDNA.

Single-stranded DNA (ssDNA) is useful for aptamers (1), self-assembly (2), DNA origami (3), DNA circuits (4), hybridization probes (5), and DNA robotics (6). Chemically synthesized DNA is single-stranded by default.

Here, we present a method to produce ssDNA from a PCR product. The use of a PCR product instead of synthetic DNA may be advantageous since PCR introduces fewer mutations than chemical synthesis and can generate DNAs longer than the -120 base limit for chemical synthesis. Starting from a clonal sample, PCR products can also have significantly higher sequence purity than chemically synthesized DNA. High sequence purity is critical for high performance in DNA circuit technology (7).

Our single-strand generation (SSG) method is scalable and requires only one purification step. This removes the unwanted complementary strand and performs sizebased purification from residual primer and unwanted PCR products. SSG by co-polymerization and electrophoresis may be applied to the product of advanced PCR protocols such as Gibson assembly (8) to create long, single-stranded products of arbitrary sequence. This may be of use for DNA origami and other self-assembly applications.
SSG takes advantage of a commercially available DNA modification known as an acrydite. This modification adds a polymerizable vinyl group, which incorporates into a growing acrylamide polymer chain (9). Acrydite-modified DNA has been used for a number of applications including a switchable hydrogel (10), capture of mercury (11) or PDGF (12), and for modifying the rate of electrophoresis of specific sequences (13). Our technique is superficially similar to the method by which the Church group immobilized polymerase colonies within thin polyacrylamide gels. The acrydite is critical for the formation of clonal PCR products (polonies) in some forms of DNA sequencing (14).

In cases when significant quantities of pure ssDNA are required, our approach using acrydite-modified primers and polyacrylamide immobilization is a worthwhile option. We also show that this technique can integrate SSG, size separation, and electroelution into a single step.

\section{Materials and methods}

Unless otherwise stated, reagents were acquired from Sigma Aldrich (St. Louis, $\mathrm{MO}$ ) and used without further purification.
DNA was acquired from IDT (Integrated DNA Technologies, Coralville, IA) and used without further purification (for sequence information, see Supplementary Table S1).

Demonstration of capture

of acrydite strand

For the initial demonstration of SSG by co-polymerization with acrylamide and then electrophoresis, we modified one primer with acrydite and Cy5 red fluorescent dye. The other primer was purchased with a green fluorescent $5^{\prime}$ fluorescein modification. Figure 1 shows how the DNA sample was prepared by mixing $100 \mu \mathrm{l} 10 \%$ denaturing gel (prepolymer before initiation of polymerization) with $100 \mu$ l PCR product ( 20 pMol of product) and the appropriate mass of dry urea (5\% final acrylamide concentration, $7 \mathrm{M}$ final urea concentration). The DNA/prepolymer was loaded into a dry well of a $5 \%$ denaturing PAGE gel. The gel was heated as described below, and electrophoresis was performed to separate the green fluorescent strand from the immobilized red fluorescent strand. The resulting gel was imaged using a standard gel imaging system with LED illumination (FluorChem Imaging System, ProteinSimple, San Jose, CA).

\section{METHOD SUMMARY}

Commercially available 5'-acrydite-modified DNA primers are used to purify single stranded DNA (ssDNA) by immobilizing one strand of a PCR product within a polyacrylamide matrix. 
Single-strand generation and

purification (vertical gel electrophoresis)

PCR was performed in all cases with the Accuprime Pfx reaction kit (Life Technologies, Grand Island, NY) using 50 nM template, 10 $\mu \mathrm{M}$ of each primer, and 8 thermal cycles. The PCR product was denatured by adding solid urea to a final concentration of $7 \mathrm{M}$ in a $100 \mu \mathrm{l}$ reaction. Polyacrylamide was prepared from a commercially obtained stock solution of $40 \%$ acrylamide in water with a 19:1 ratio of acrylamide:bis-acrylamide (Bio-Rad, Hercules, CA). Denaturing polyacrylamide was prepared with final concentrations of $7 \mathrm{M}$ urea, $20 \%$ acrylamide, and 0.25x TBE buffer. Polymerization was initiated by adding $1 \mu \mathrm{l}$ of TEMED (N,N,N'N'N-tetramethylethylenediamine) and 10 $\mathrm{\mu l} 10 \%$ ammonium persulfate to an aliquot of $1 \mathrm{~mL}$ of denaturing acrylamide prepolymer. A portion of this mixture (prepolymer before initiation of polymerization) was rapidly mixed

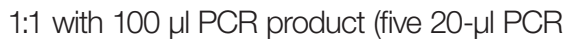
reactions per manufacturer's instructions, pooled) with $7 \mathrm{M}$ urea (10\% acrylamide, final concentration). The PCR/polymerizing acrylamide mixture was added to a dry well in a standard vertical denaturing PAGE gel. Full polymerization of the PCR/denaturing acrylamide was ensured by flushing the space above the well with a gentle stream of argon or $99.97 \%$ nitrogen (to displace any oxygen that inhibits the polymerization). After $\sim 30 \mathrm{~min}$ of polymerization, we set up the electrophoresis rig. Crushing the polyacrylamide and loading the macerated pieces into the well produced a very broad, irregularly shaped band, so this approach was abandoned (data not shown). Polymerization within the well was a superior approach. We heated running buffer in a microwave to boiling and added the hot $\left(\sim 80^{\circ} \mathrm{C}-90^{\circ} \mathrm{C}\right)$ buffer to the cathode reservoir (by pouring the hot buffer over the filled wells) in order to encourage release of the DNA from the well. The addition of the hot buffer was continued until the gel rig was full to the necessary volume for electrophoresis. The DNA-containing gel was in direct contact with hot buffer as voltage was applied. The hot buffer should be applied promptly and not be allowed to cool. We then performed electrophoresis according to the diagram shown in Figure 1.

SB (5 mM sodium borate) and TBE buffers can be used as running buffers. SB and TBE were prepared at $\mathrm{RT}$ at $\mathrm{pH} 8.42$ and 8.36 respectively. At $80^{\circ} \mathrm{C}$, these $\mathrm{pH}$ values change to 8.21 and 7.47 respectively. This $\mathrm{pH}$ change is transient. The gel cools to $30^{\circ} \mathrm{C}-40^{\circ} \mathrm{C}$ during electrophoresis. This change in $\mathrm{pH}$ did not cause noticeable degradation of the DNA.

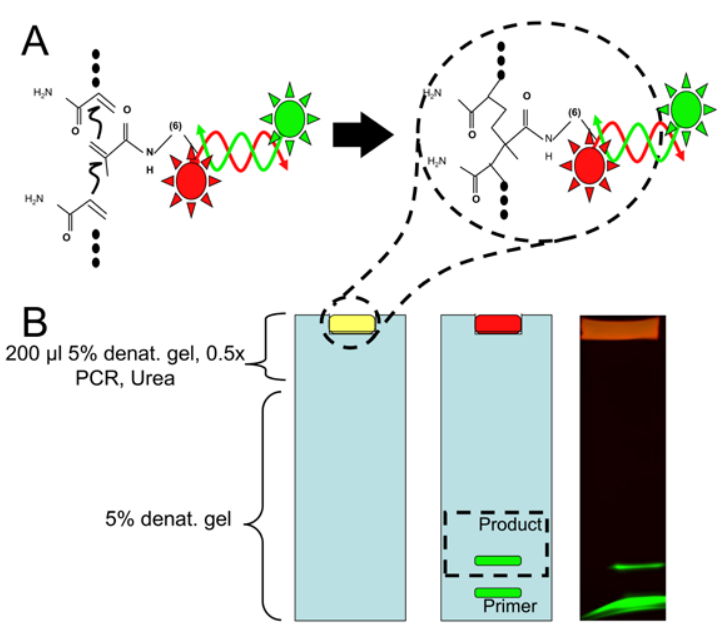

Figure 1. Outline of the single-strand generation (SSG) technique. (A) The schematic shows how double-stranded DNA (dsDNA) is produced from a primer modified with acrydite and $\mathrm{Cy} 5$ at its $5^{\prime}$ terminus and a reverse primer modified with fluorescein. These primers produce a double-stranded PCR product. When co-polymerized with acrylamide, this product is immobilized within the polymer matrix. (B) Fluorescence gel image (right) shows that the Cy5-modified, co-polymerized strand remains at the top of the gel, while the single-stranded product and primer migrate separately.

In order to visualize the separation of the two strands, we used primers modified with two different fluorophores. The mobile strand was generated using a fluorescein-modified primer. The primer (acrydite) for the immobile strand was prepared with an internal Cy5 modification. Using these two dyes, we could visualize the progress of the separation. We scanned this gel with a gel imager (FluorChem Q, ProteinSimple, Santa Clara, CA) after 45 min of electrophoresis at $500 \mathrm{~V}$.

As an alternative approach, the PCR product can be pre-concentrated by standard ethanol precipitation. The pellet can then be dissolved in a smaller volume of acrylamide prepolymer. In our hands, the increased concentration in the well was offset by losses during precipitation. In some cases (e.g., for a faint product band) this approach may be preferred.

The fluorescent band bearing the singlestranded, fluorescein-modified product was cut from the gel under blue LED illumination at $475 \mathrm{~nm}$ (Bulldog Bio, Portsmouth, $\mathrm{NH}$ ). The DNA was eluted by the standard crush and soak method (15). When the eluted (recovered) DNA was too dilute for efficient ethanol precipitation, we concentrated it by extraction with butanol. The product was then precipitated with ethanol and analyzed further as indicated below.

\section{PAGE analysis}

The fluorescein-labeled single-stranded products (ssDNA released by denaturing PAGE) were analyzed for purity using native PAGE. We resuspended the single-stranded product in $10 \mu \mathrm{l}$ and quantified it by UV-Vis spectroscopy. We made equal molar concentrations of primer and single-stranded product and electrophoresed these samples and a 25-bp green fluorescent ladder (Jena Bioscience, Jena, Germany) on a 15\% native
PAGE gel, which was visualized with the Storm scanner for the fluorescein signal.

\section{Quencher analysis}

To further establish that the product was in fact single-stranded, we tested for the hybridization of a complementary oligonucleotide (quench probe) containing a 3' quencher modification. When properly hybridized, the quench probe positions an lowa Black quencher within a few nanometers of the 5 fluorescein modification on the complementary strand. This causes a sharp decrease in fluorescence intensity. The quench probe will only hybridize to ssDNA. If the product is double-stranded, then hybridization will be blocked, and fluorescence will be unaffected. Triplicate $10 \mu \mathrm{l}$ samples of $100 \mathrm{nM}$ fluoresceinmodified products (PCR product, SSG product, and primer control) were prepared in PCR tubes. These were measured with a QuantiFluor fluorometer (Promega, Madison, $\mathrm{WI}$ ) for the initial fluorescence values, and 1.1 molar equivalents of quench probe DNA were then added, vortexed, centrifuged, and allowed to incubate for approximately 1 min. The fluorescence was then recorded to determine if any quenching occurred.

Comparison of single-strand generation techniques for aptamer production

In order to ascertain the effectiveness of this method for generating functional aptamers, we purchased a template for PCR amplification of a known aptamer sequence binding lysozyme. We amplified this template with a fluorescein-modified primer and an acryditemodified reverse primer. We performed the SSG protocol as described above. We conjugated lysozyme to $5.8 \mu \mathrm{m}$ carboxylatemodified beads (Bangs Labs, Fishers, IN) by the standard EDC [1-ethyl-3-(3-dimethylaminopropyl) carbodiimide] coupling protocol. These 
beads were incubated with the SSG product, fluorescent randomized DNA (no sequence similarity to the aptamer), and a chemically synthesized fluorescent aptamer of the same sequence. Wealso incubated uncoated beads with the chemically synthesized fluorescent aptamer as a negative control. Incubations were carried out for 30 min and the beads were then washed 3 times. Flow cytometry was performed on these particles with a FACScalibur (BD Bioscience San Jose, CA). The distribution of fluorescence intensities with excitation by 488-nm light was recorded.

Single-strand generation and purification (horizontal gel electrophoresis)

A $7 \mathrm{M}$ urea denaturing polyacrylamide gel was cast horizontally as described previously (16). Briefly, $25 \mathrm{~mL}$ prepolymer solution (7M urea, $6 \%$ acrylamide/bis-acrylamide, SB buffer) was initiated with $84 \mu \mathrm{I}$ APS and $20 \mu \mathrm{ITEMED}$. The gel prepolymer was poured into a horizontal mold. The mold was then placed in a plastic bag, which was purged with nitrogen. The gel was allowed to polymerize for $30 \mathrm{~min}$. Fluorescein-labeled and acrydite-labeled DNAs were mixed in $1 \mathrm{M}$ sodium phosphate at $\mathrm{pH} 8.0$ and annealed by raising the temperature to $80^{\circ} \mathrm{C}$ and cooling slowly at $0.1^{\circ} \mathrm{C}$ per second to RT. The mixture was cooled slowly to promote intermolecular hybridization between two strands. The prepolymer mixture (2.5 mL) was initiated by adding $8.4 \mu \mathrm{l}$ APS and $2 \mu \mathrm{l}$ TEMED. Once mixed, $20 \mu$ of this solution were added to the $5 \mu \mathrm{l}$ DNA solution. This was then transferred to a dry well in the polyacrylamide gel. In order to avoid distortions in the electric field lines, the remaining wells were filled with non-DNA-containing acrylamide. The loaded gel was placed in a plastic bag and purged with nitrogen. The gel was run at $10 \mathrm{~V} / \mathrm{cm}$ for $10 \mathrm{~min}$. It was then imaged using a blue LED transilluminator and a digital camera. In order to release the ssDNA from the crosslinked gel in the well, $25 \mathrm{~mL}$ SB buffer was heated to boiling in a microwave. This hot buffer was then poured over the gel. Electrophoresis was continued at $10 \mathrm{~V} / \mathrm{cm}$ for $10 \mathrm{~min}$. The gel was imaged again as described above for comparison.

Initial association between the fluoresceinand acrydite-labeled DNA was promoted using $1 \mathrm{M}$ sodium phosphate buffer. If the DNA were only held together by hybridization, $7 \mathrm{M}$ urea and the exchange of buffer into 5 $\mathrm{mM}$ SB buffer during electrophoresis would be adequate to release the DNA into the gel. Instead, heat was required. This demonstrated that the mobile DNA strand is associated or

A
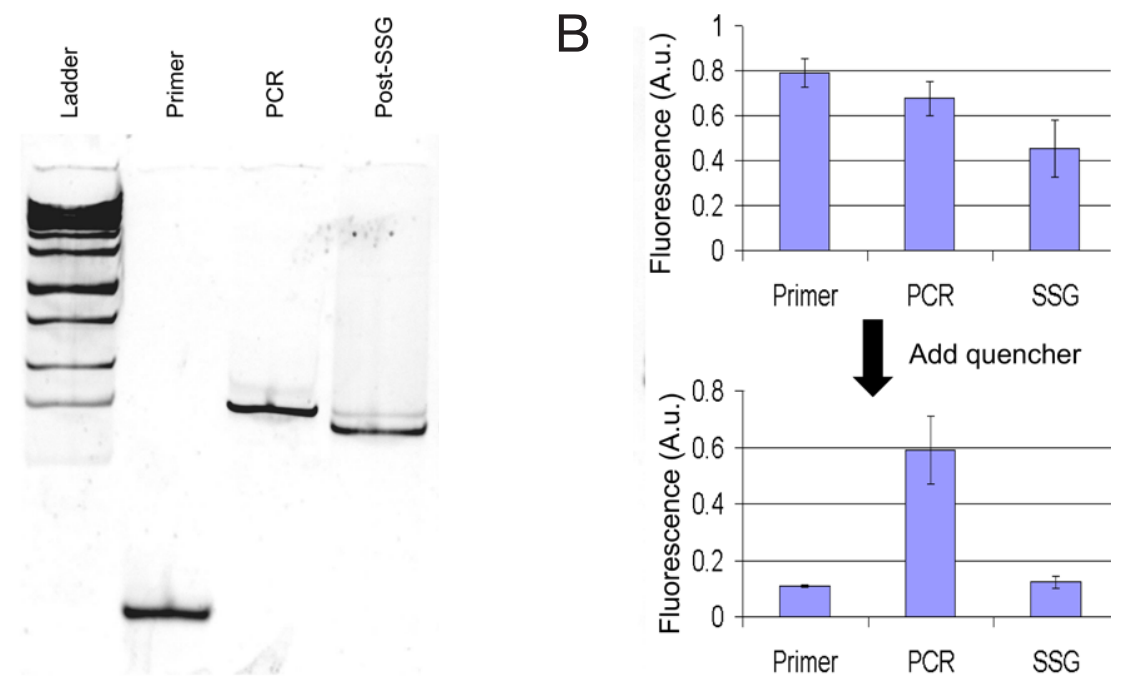

Figure 2. Verification of single-stranded DNA (ssDNA) purification. (A) Native gel image shows clear resolution of a single-strand generation (SSG) product as compared with a double-stranded DNA (dsDNA) PCR product. (B) Fluorescence intensity shows the result of FRET-based quenching using a hybridization probe to bind ssDNA.

entangled with the gel rather than with its complementary DNA strand.

\section{Results and discussion}

Acrydite-modified DNA is immobile under electrophoresis Of the two strands of the dsDNA PCR product, the acrydite strand is trapped in the co-polymer, while the complementary strand is mobile. To demonstrate this, PCR was performed with one primer modified with both acrydite and a red fluorescent dye (Cy5). The reverse primer was modified with fluorescein. The PCR product was polymerized into a denaturing PAGE gel (5\%). A schematic diagram of the process is presented in Figure 1. Initially, both the green fluorescent and red fluorescent strands are co-localized (yellowcolored gel). Upon application of voltage, only the fluorescein-labeled product (green) migrates into the gel. The acrydite and Cy5 co-labeled strand (red) is fixed in the final gel image (Figure 1B). Additionally, residual primer from the PCR reaction is clearly visible as a second green band. After electrophoresis, the pure, single-stranded product can be cut and eluted per standard gel purification protocols.

\section{Single-stranded DNA verification}

We isolated single-stranded, fluoresceinmodified DNA by cutting and eluting the band of interest. To show that the product recovered by this method is single-stranded, we used two different methods. The first method was native gel analysis. Figure 2A shows an image of a native gel containing the primer, PCR product (dsDNA), and ssDNA purified by this method. The native gel shows clear separation between the dsDNA and ssDNA. After SSG with the acrydite primer (followed by cutting the gel and eluting the product) most of the isolated DNA is single-stranded.

We corroborated this result using a quencher-modified hybridization probe (Figure 2B). The probe was designed to bring a quencher into proximity of the fluorescein moiety on the ssDNA product. Upon hybridization, the quencher molecule reduces fluorescence intensity by 90\%. As the probe only hybridizes to ssDNA, this experiment demonstrates that the fluorescein-modified product was single-stranded. As a negative control, we also tested the dsDNA PCR product. The double-stranded PCR product was unable to hybridize to the quencher probe; therefore, its fluorescence remained virtually unchanged.

\section{Aptamers synthesized by PCR purified by co-polymerization and electrophoresis}

Highly pure ssDNA is produced by our SSG technique. We purified ssDNA aptamer products from PCR using two methods: (i) SSG by co-polymerization and electrophoresis and (ii) a biotin-avidin immobilization technique described elsewhere (i.e., Polysciences Technical data sheet 753) $(17,18)$. Single-stranded aptamers were also generated by chemical synthesis. Figure $3 \mathrm{~A}$ shows ssDNA aptamers generated using the three different methods. Lane 1 is a marker ladder for size comparison. Lane 2 shows the purified SsDNA generated by PCR with an acrydite primer followed by co-polymer- 
ization with acrylamide, electrophoresis, and gel purification. Only one band is visible at the correct size for ssDNA. Lane 3 is the ssDNA generated by PCR with a biotinylated primer followed by purification with avidin-coated magnetic beads. Lane 4 is chemically synthesized DNA with the same sequence. Purified ssDNA aptamer from SSG by co-polymerization and electrophoresis showed high ssDNA purity.

We next sought to test if this procedure produced functional aptamers. To do so, we generated a previously described aptamer against lysozyme using PCR with modified primers. The "Clone 1" anti-lysozyme aptamer was first produced in the Ellington laboratory and was originally selected as an RNA aptamer (19); other groups have reported that the DNA sequence also acts as an aptamer $(20,21)$. We found that both PCR and chemical DNA synthesis produced a functional aptamer. Based on these and other experiments, we conclude that this is one of the few, exceptional cases where RNA and its parent DNA sequence both bind the target. This is a strange and coincidental result; most aptamers do not function when translated directly to a different chemistry. These results corroborate the many instances in the literature indicating that the Clone 1 anti-lysozyme aptamer is a special case.

Flow cytometry analysis also showed that the SSG-purified ssDNA aptamer is functional. Figure 4B shows lysozyme-coated beads exposed to random DNA, the chemically synthesized aptamer against lysozyme, or our SSG-purified aptamer against lysozyme. Both the SSG-purified and chemically synthesized aptamers showed strong binding to the lysozyme-coated beads, whereas the random DNA showed no significant binding to the lysozyme beads. This indicates that these interactions are specific for the aptamer sequence. Uncoated beads show no fluorescence with or without the aptamer, indicating that the binding is specific to the protein.

Heating releases DNA from wells When purifying ssDNA aptamers from the PCR product using the SSG method, it was necessary to heat the gel to facilitate DNA electrophoresis (see "Materials and methods"). To understand why this was necessary, we used horizontal PAGE. Without the application of heat, the desired ssDNA PCR product was retained in the well, despite the use of $7 \mathrm{M}$ urea and the voltage gradient.

Our first hypothesis was that this retention was due to the 80 bp of hybridization in the
PCR product. To test this theory, we reduced the complementarity to 23 bp by annealing the fluorescein-labeled Clone 1 aptamer DNA (denoted F-Aptamer in Figure 4) to a 23-nucleotide-long acrydite-modified primer (denoted AC-Clone1- $P_{2}$ in Figure 4) and performed SSG by co-polymerization and electrophoresis. Despite the relatively short 23 bp hybridization, significant quantities of the aptamer DNA were retained in the well (Figure 4A, bottom lane). This indicated that our hypothesis was incorrect since the shorter hybridization stretch of $23 \mathrm{bp}$, which should be easily denatured in 7 M urea, did not allow the release of the desired ssDNA strand from the well.

We next tested the hypothesis that retention was dependent on length. Here, we annealed a 5'-acrydite modified 19-nucleotide-long oligonucleotide (denoted AC-DNA in Figure 4) to a 19-nucleotide fully complementary, fluorescein-labeled oligonucleotide (denoted F-DNA* in Figure 4). Despite the similar hybridization length, the short DNA was easily purified from its complement in a denaturing gel without the application of heat. Virtually all of the 19-nucleotide, fluoresceinlabeled mobile strand enters the gel (Figure $4 \mathrm{~A}$, middle lane). In order to show that the acrydite-modified 19-mer was retained, a strand modified with both fluorescein and acrydite (denoted AC-DNA-F in Figure 4) was also co-polymerized into the well (top lane) and

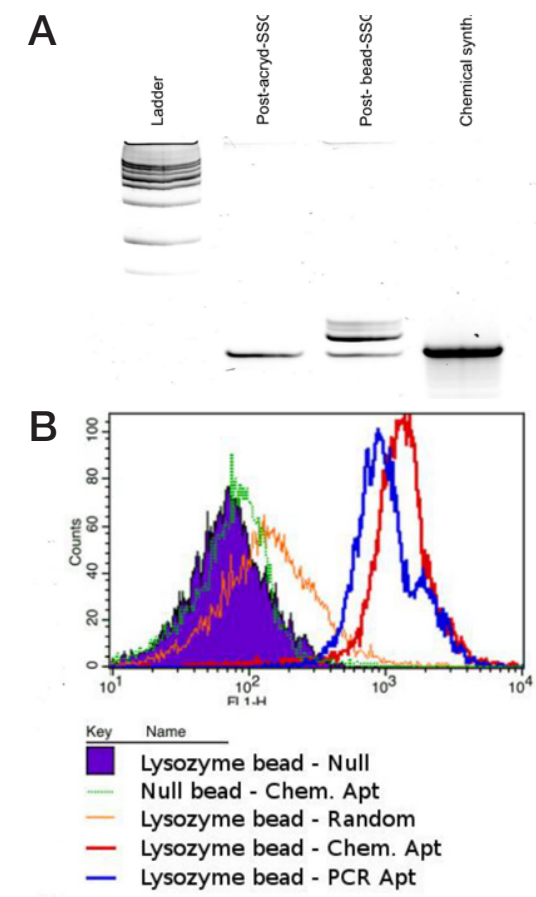

Figure 3. Purity and functionality of aptamers derived from our single-strand generation (SSG) method and other techniques. (A) Native gel analysis shows the purity of SSG by co-polymerization and electrophoresis, SSG using avidin-coated beads, and chemical synthesis. (B) Flow cytometry shows relative fluorescence of lysozyme beads (purple), lysozyme beads with random DNA (orange), uncoated beads mixed with synthetic aptamer (green), lysozyme beads mixed with synthetic aptamer (red), and lysozyme beads mixed with aptamer derived from PCR and SSG by co-polymerization and electrophoresis (blue).

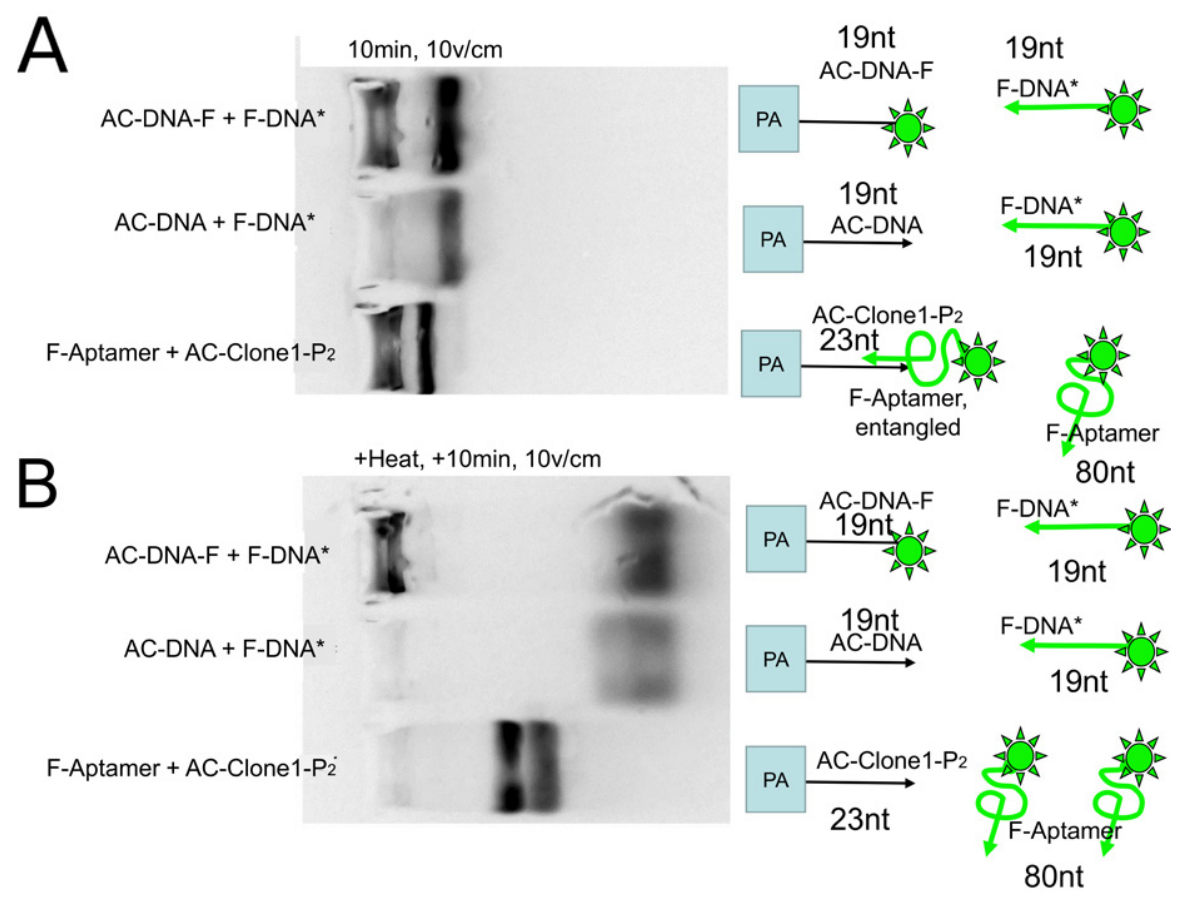

Figure 4. PAGE analysis shows that added heat is required for successful single-strand generation (SSG). (A) Fluorescence image of gel after $10 \mathrm{~min}$ of electrophoresis. Schematic at right shows the contents of each well and the corresponding bands. (B) Fluorescence image of the same gel after applying heat, followed by 20 min of further electrophoresis. Heat released all of the long DNA (third lane). 
was successfully retained. (see "Materials and methods" and Supplementary Table S1 for sequence and nomenclature details)

After the first 20 min of electrophoresis (without heating), it was clear that much of the Clone 1 aptamer DNA was not mobile (despite a short hybridization length). In order to release the aptamer DNA from the polyacrylamide, it was necessary to heat the gel. We heated running buffer to near boiling in a microwave and then poured it over the gel in the region of the wells. Electrophoresis was then continued for an additional $20 \mathrm{~min}$ (Figure 4B, bottom lane), and the aptamer band then became mobile. This new band had the same mobility as the initial mobile band, indicating that this was the same aptamer species. Likewise, this was the same sample of DNA that yielded only a single band in Figure 3A. This experiment demonstrates the necessity of heating the gel in order to release all long ssDNA from the polyacrylamide. The retention of DNA in the well is not due to hybridization but likely to entanglement in the polyacrylamide matrix.

\section{Horizontal PAGE for single-strand} generation and integrated electroelution Use of a horizontal gel allows for electroelution to be carried out rather than cutting and extracting the product band. The use of a second extraction comb for additional wells for electroelution and extraction in horizontal PAGE made SSG and electroelution of DNA faster. We used Inkscape software to design a separate comb for electroelution that was deeper and wider than the loading comb (see Supplementary Material for the template used to cut the combs). The design was laser-cut in acrylic with a $\mathrm{CO}_{2}$ laser cutter (see Figure 5, A and B).

Typically, PAGE is carried out using a vertical gel between glass plates. To cast a horizontal PAGE gel, it was necessary to exclude oxygen during polymerization. We carried out the polymerization in a bag filled with nitrogen gas (or argon) to exclude oxygen. The gel was cast with the two combs, and electrophoresis was carried out per standard horizontal gel electrophoresis procedures.

Figure 5C shows a horizontal gel for SSG by co-polymerization and electrophoresis with electroelution. Lanes 1-4 (from top) contain an aptamer pool. Lane 5 was left empty to avoid any inadvertent cross-contamination; primer standard in Lane 6 was present to distinguish the undesired band. The pool was amplified using an acrydite-modified primer and a fluorescein-labeled primer. The acryditemodified strand was retained in the well. The
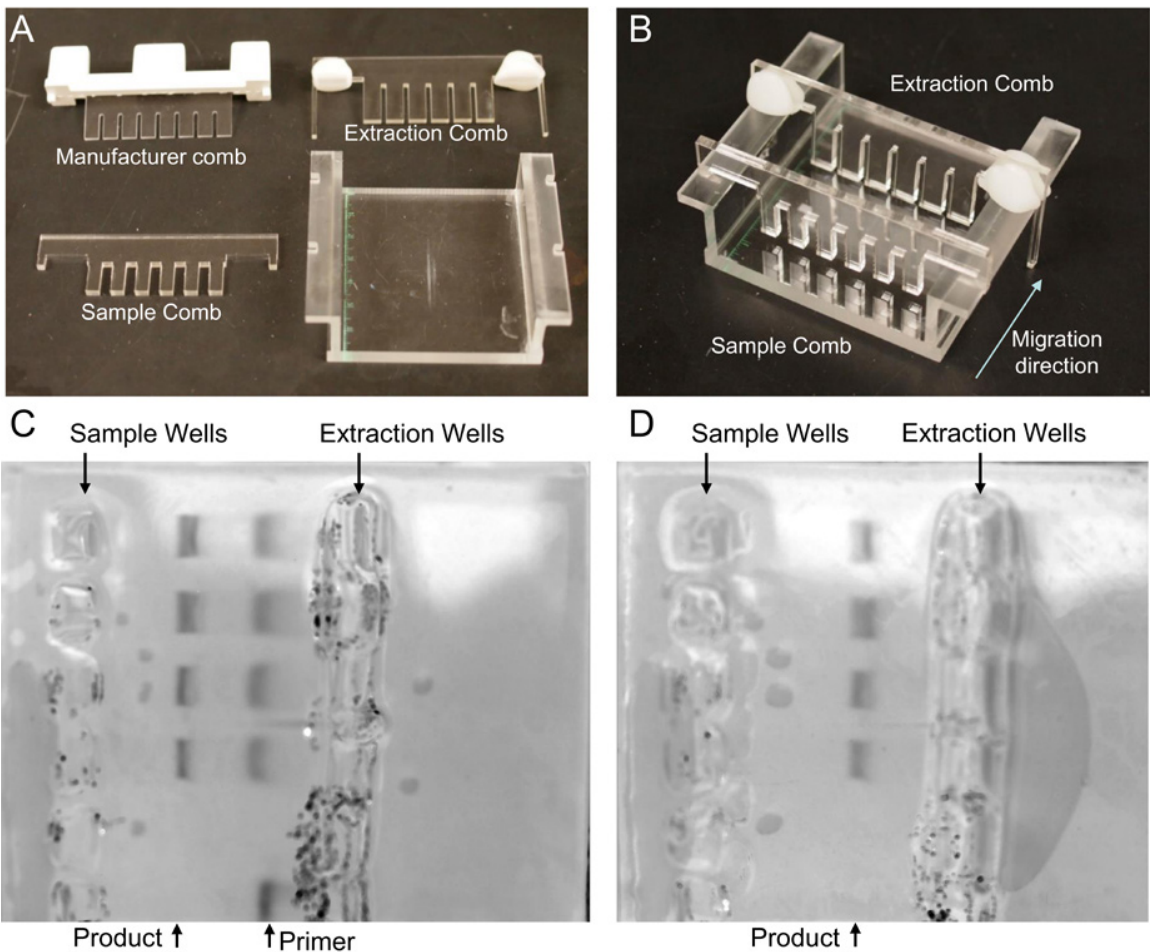

Figure 5. Demonstration of electroelution technique using a set of extraction wells molded into a horizontal polyacrylamide gel. (A) Photograph showing the original sample comb and our adapted injection and extraction combs, as well as the Bio-Rad gel tray for which they were designed. (B) Photograph showing the extraction and injection combs in the tray for molding. (C) Fluorescence image showing the separation of product and primer from a PCR-amplified aptamer pool. (D) Fluorescence image showing the same gel after further separation, with the product poised to enter the extraction wells for recovery.

fluorescein-labeled product is clearly visible under blue LED illumination. We allowed the primer to run through the extraction wells and out the other side of the gel (see Figure 5D). The product bands could then be observed approaching the extraction wells under a blue light transilluminator in real time. When the product bands entered the wells, they were aspirated with a pipette. The process took approximately $1 \mathrm{~h}$. Precipitation and recovery then proceeded as per published aptamerselection protocols. This eliminated the need for a separate strand-separation step or an overnight extraction of the gel.

There are several methods for isolating ssDNA from dsDNA. Methods for purifying ssDNA from PCR products include: induced mobility shift (22), biotin-avidin bead immobilization (18), selective digestion with a DNase (23), and asymmetric addition of PCR primers (24). These methods vary in cost, purity and scalability. An induced mobility shift (22) in one primer can cause problems in amplification (e.g., PEG-modified primers may perform less well in PCR), and enzymatic digestion with a DNase (23) or chemical breakage of one strand will leave impurities (as well as introducing another step to the overall process). Asymmetric addition of PCR primers (24) will leave a significant quantity of dsDNA in the product and is highly prone to amplification errors. The most popular method is to extract the unwanted, biotinylated strand using avidin-coated microspheres (18). This method has several pitfalls. Unreacted primer occupies the avidin sites on the beads, and the biotin-avidin interaction will break down at the melting temperature of long, doublestranded DNA (25). This contributes to significant dsDNA impurities. Bead-based methods have significant costs: $\$ 1.80 / \mathrm{nMol}$ for avidin beads plus $\$ 0.50 / \mathrm{nMol}$ for the biotin primer. SSG by co-polymerization and electrophoresis requires only acrydite primer, which costs $\$ 0.80$ per $\mathrm{nMol}$. Polyacrylamide is very inexpensive and scalable. Electrophoretic mobility is a second dimension of purification inherent in the technique. Both the acryditemodified strand and any residual primer are removed from the PCR reaction in a single step.

We have shown that SSG can be used to purify a functional aptamer. The technique could also be used to purify an ssDNA pool in the course of DNA aptamer selection. In addition, SSG may also find application for generating ssDNA for affinity probes or backbones for DNA origami (3). It's important to note that compared with chemical synthesis, PCR 
production of DNA has much higher sequence fidelity, which is crucial for applications such as DNA computation/DNA circuitry (26).

\section{Author contributions}

P.B.A. and T.R.D wrote the main manuscript text, prepared figures, and conducted experiments. A.D.E. advised on aptamer applications and verification, and helped prepare and edit the manuscript.

\section{Acknowledgments}

This work was funded by the National Institutes of Health (EUREKA, 1-R01-GM094933), The Welch Foundation (F-1654), a National Security Science and Engineering Faculty Fellowship (FA9550-10-1-0169), and support from the National Institute of General Medical Sciences (P20-GM104420). This paper is subject to the NIH Public Access Policy.

\section{Competing interests}

The authors declare no competing interests.

\section{References}

1. Cho, E.J., J.-W. Lee, and A.D. Ellington. 2009. Applications of Aptamers as Sensors. Annu Rev Anal Chem (Palo Alto Calif). 2:241-264.

2. Tang, H., R. Deschner, P. Allen, Y. Cho, P. Sermas, A. Maurer, A.D. Ellington, and C.G. Willson. 2012. Analysis of DNA-Guided Self-Assembly of Microspheres Using Imaging Flow Cytometry. J. Am. Chem. Soc. 134:15245-15248.

3. Rothemund, P.W.K. 2006. Folding DNA to create nanoscale shapes and patterns. Nature 440:297302.

4. Allen, P.B., S.A. Arshad, B. Li, X. Chen, and A. Ellington. 2012. DNA circuits as amplifiers for the detection of nucleic acids on a paperfluidic platform. Lab Chip 12:2951-2958.

5. lida, K. and I. Nishimura. 2002. Gene Expression Profiling by DNA Microarray Technology. Crit. Rev. Oral Biol. Med. 13:35-50.

6. Olah, M.J. and D. Stefanovic. 2011. Multivalent Random Walkers - A model for deoxyribozyme walkers, p. 160-174. In L. Cardelli and W. Shih (Eds), DNA Computing and Molecular Programming, Springer Verlag, Berlin, Germany.

7. Chen, X., N. Briggs, J.R. McLain, and A.D. Ellington. 2013. Stacking nonenzymatic circuits for high signal gain. Proc. Natl. Acad. Sci. USA 110:5386-5391.

8. Gibson, D.G., G.A. Benders, C. AndrewsPfannkoch, E.A. Denisova, H. Baden-Tillson, J. Zaveri, T.B. Stockwell, A. Brownley, et al. 2008. Complete Chemical Synthesis, Assembly, and Cloning of a Mycoplasma genitalium Genome. Science 319:1215-1220.

9. Kenney, M., S. Ray, and T.C. Boles. 1998. Mutation typing using electrophoresis and gel-immobilized Acrydite probes. BioTechniques 25:516-521.

10. Liedl, T., H. Dietz, B. Yurke, and F. Simmel. 2007. Controlled Trapping and Release of Quantum Dots in a DNA-Switchable Hydrogel. Small 3:1688-1693.
11. MacLean, J.L., K. Morishita, and J. Liu. 2013. DNA stabilized silver nanoclusters for ratiometric and visual detection of $\mathrm{Hg} 2+$ and its immobilization in hydrogels. Biosens. Bioelectron. 48:82-86.

12. Soontornworajit, B., J. Zhou, M.T. Shaw, T.-H. Fan, and Y. Wang. 2010. Hydrogel functionalization with DNA aptamers for sustained PDGF-BB release. Chem. Commun. (Camb.) 46:1857-1859.

13. Kenney, M., S. Ray, and T.C. Boles. 1998. Mutation typing using electrophoresis and gel-immobilized Acrydite probes. BioTechniques 25:516-521.

14. Mitra, R.D. and G.M. Church. 1999. In situ localized amplification and contact replication of many individual DNA molecules. Nucleic Acids Res. 27:e34-e39.

15. Chen, Z. and D.E. Ruffner. 1996. Modified crushand-soak method for recovering oligodeoxynucleotides from polyacrylamide gel. BioTechniques 21:820-822.

16. Damase, T.R., D. Stephens, A. Spencer, and P.B. Allen. 2015. Open source and DIY hardware for DNA nanotechnology labs. J. Biol. Methods 2:e24.

17. Liang, C., D. Li, G. Zhang, H. Li, N. Shao, Z. Liang, L. Zhang, A. Lu, and G. Zhang. 2015. Comparison of the methods for generating single-stranded DNA in SELEX. Analyst. 140:3439-3444.

18. Kuo, T.-C. 2005. Streamlined method for purifying single-stranded DNA from PCR products for frequent or high-throughput needs. BioTechniques. 38:700702.

19. Kirby, R., E.J. Cho, B. Gehrke, T. Bayer, Y.S. Park, D.P. Neikirk, J.T. McDevitt, and A.D. Ellington. 2004. Aptamer-Based Sensor Arrays for the Detection and Quantitation of Proteins. Anal. Chem. 76:4066-4075

20. Rodriguez, M.C., A.-N. Kawde, and J. Wang 2005. Aptamer biosensor for label-free impedance spectroscopy detection of proteins based on recognition-induced switching of the surface charge. Chem Commun (Camb). 34:4267-4269.

21. Cheng, A.K.H., B. Ge, and H.-Z. Yu. 2007. Aptamerbased biosensors for label-free voltammetric detection of lysozyme. Anal. Chem. 79:5158-5164.

22. Pagratis, N.C. 1996. Rapid Preparation of Single Stranded DNA from PCR Products by Streptavidin Induced Electrophoretic Mobility Shift. Nucleic Acids Res. 24:3645-3646.

23. Avci-Adali, M., A. Paul, N. Wilhelm, G. Ziemer, and H.P. Wendel. 2009. Upgrading SELEX Technology by Using Lambda Exonuclease Digestion for SingleStranded DNA Generation. Molecules 15:1-11.

24. Gyllensten, U.B. and H.A. Erlich. 1988. Generation of single-stranded DNA by the polymerase chain reaction and its application to direct sequencing of the HLA-DQA locus. Proc. Natl. Acad. Sci. USA 85:7652-7656.

25. Holmberg, A., A. Blomstergren, O. Nord, M. Lukacs, J. Lundeberg, and M. Uhlén. 2005. The biotin-streptavidin interaction can be reversibly broken using water at elevated temperatures. Electrophoresis 26:501-510.

26. Yin, P., H.M.T. Choi, C.R. Calvert, and N.A. Pierce. 2008. Programming biomolecular self-assembly pathways. Nature 451:318-322.

Received 14 December 2016; accepted 11 April 2017.

Address correspondence to Peter B Allen, University of Idaho, Dept. of Chemistry, 001 Renfrew Hall, 875 Perimeter Dr, Moscow, ID 83844-2343. E-mail: pballen@uidaho.edu

To purchase reprints of this article, contact: biotechniques@fosterprinting.com 